\title{
Electrospun Polymer Composite Membrane with Superior Thermal Stability and Excellent Chemical Resistance for High-Efficiency PM2.5 Capture
}

Xue Yang, Yi Pu, Shuxia Li, Xiaofang Liu, Zheshan Wang, Ding Yuan, * Xin Ning

Industrial Research Institute of Nonwovens \& Technical Textiles, College of Textiles

\& Clothing, Qingdao University, Qingdao 266071, Shandong, People's Republic of China

Corresponding Author E-mail: * yuanding@qdu.edu.cn. 


\section{Supporting Information}
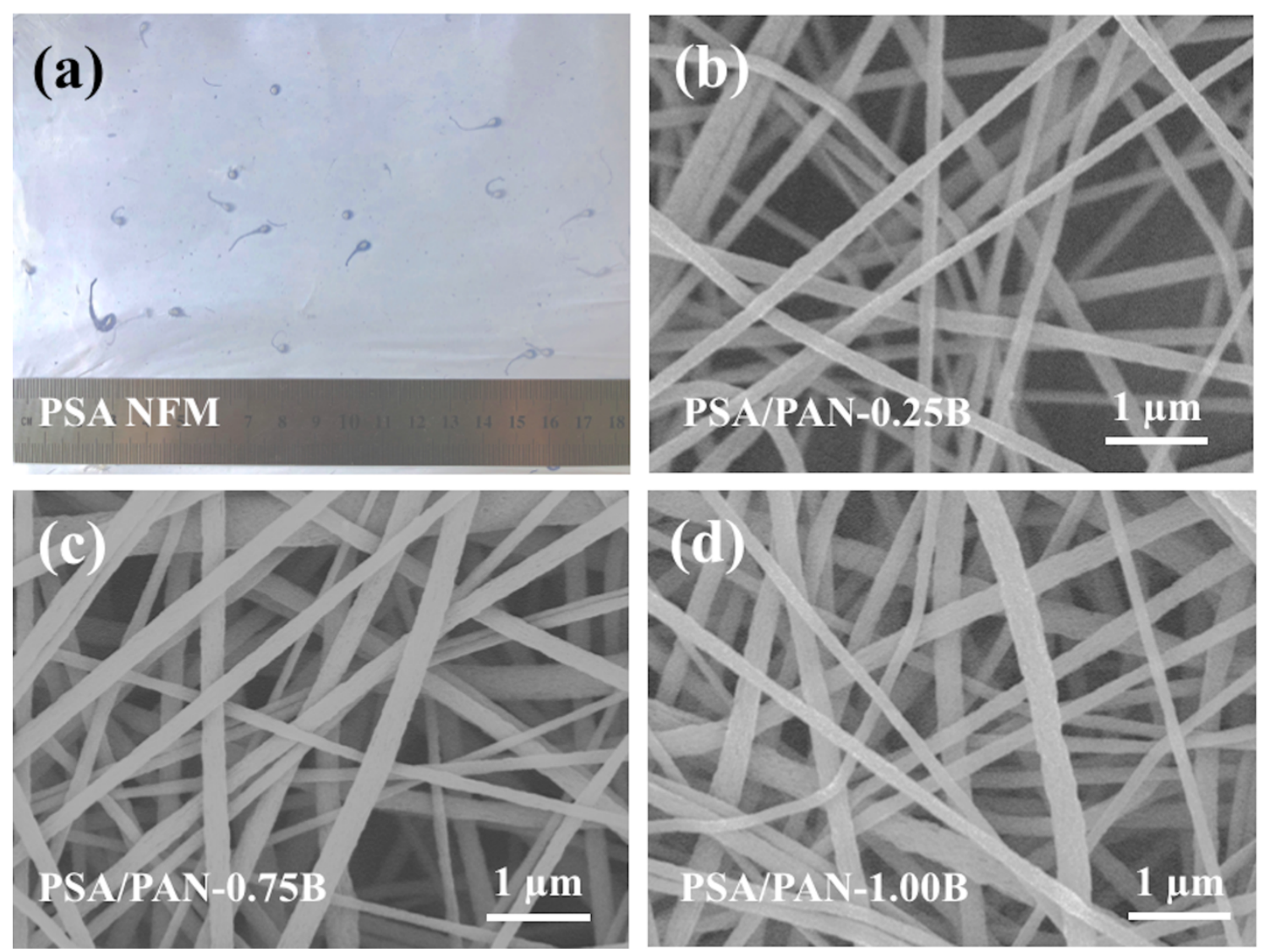

Figure S1. (a) Photography of PSA NFMs with splash defects; SEM images of PSA/PAN-xB NFMs with (b) $0.25 \mathrm{wt} \%$, (c) $0.75 \mathrm{wt} \%$, and (d) $1.00 \mathrm{wt} \%$ boehmite NPs.
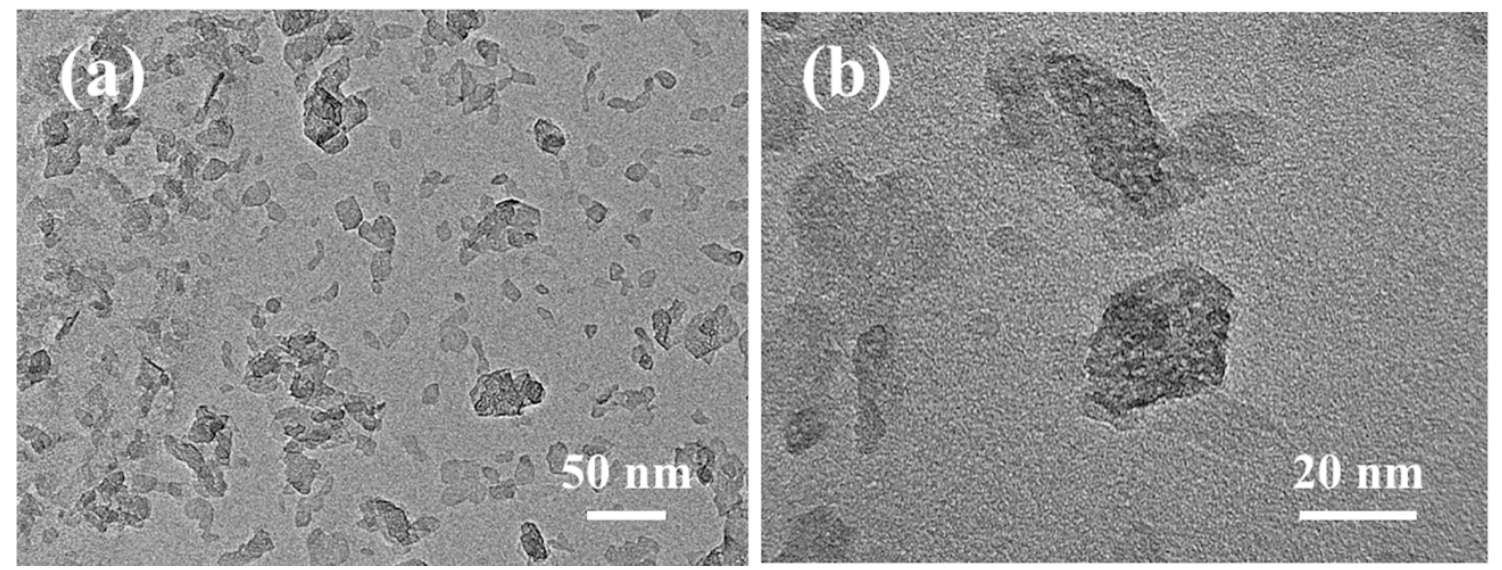

Figure S2. TEM image of individual Boehmite NPs. 


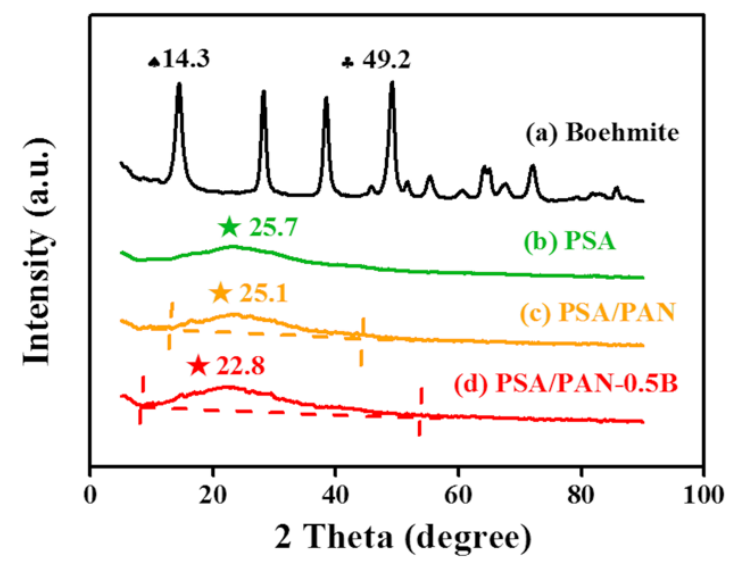

Figure S3. XRD patterns of boehmite NPs, PSA, PSA/PAN and PSA/PAN-xB NFMs.

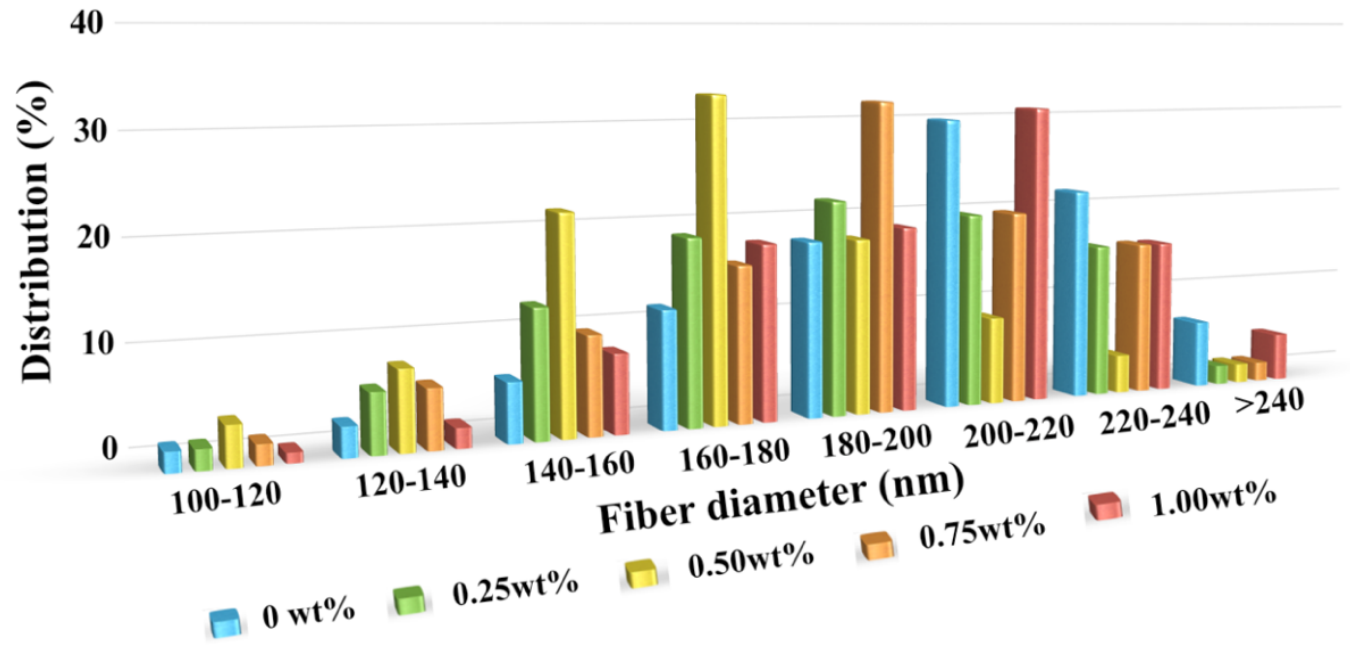

Figure S4. Fiber diameter distribution of PSA/PAN-xB NFMs.

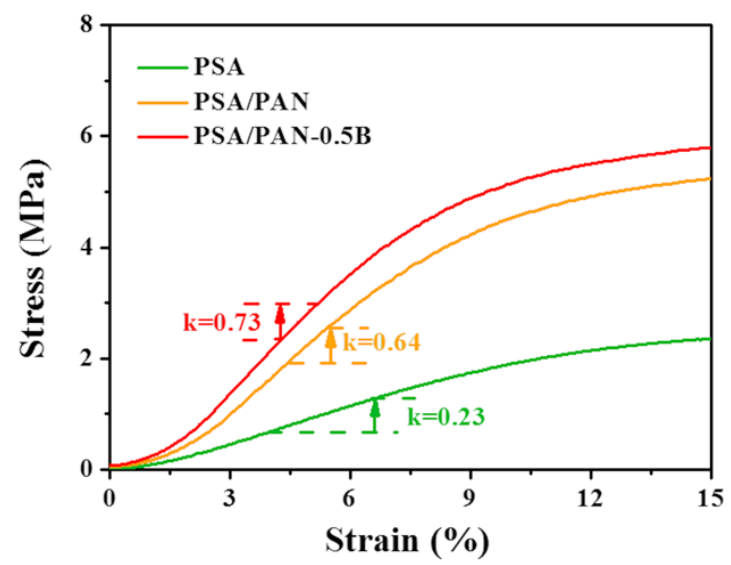

Figure S5. Young's modulus calculation of PSA, PSA/PAN and PSA/PAN-0.5B NFMs. 


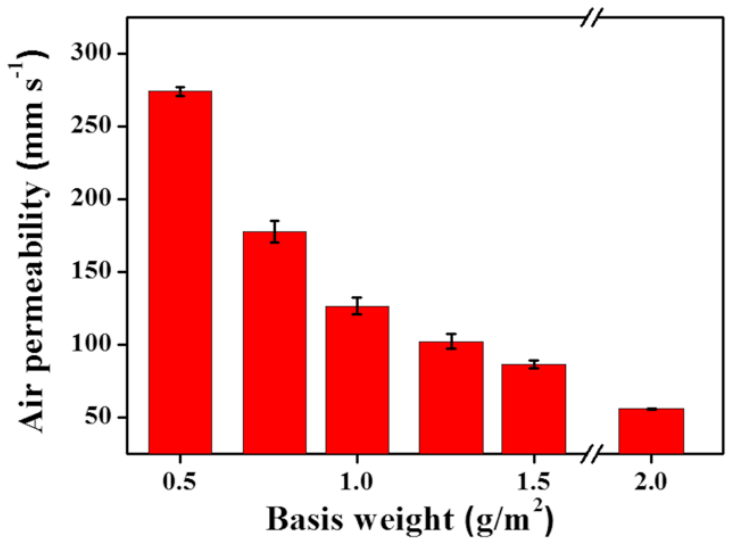

Figure S6. Air permeability of PSA/PAN-0.5B NFMs with different basis weight.

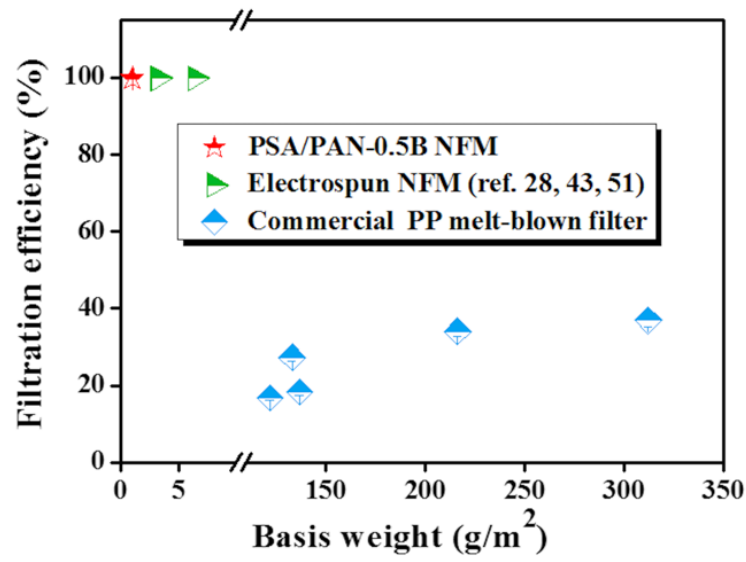

Figure S7. Air filtration comparison of PSA/PAN-0.5B NFMs, literature-based electrospun NFMs and commercial PP melt-blown filters of different basis weights. (ACS. Appl. Mater. Inter. 2016, 8, 23985; J. Mater. Chem. A. 2015, 3, 23946; ACS. Appl. Mater. Inter. 2016, 8, 8086) 

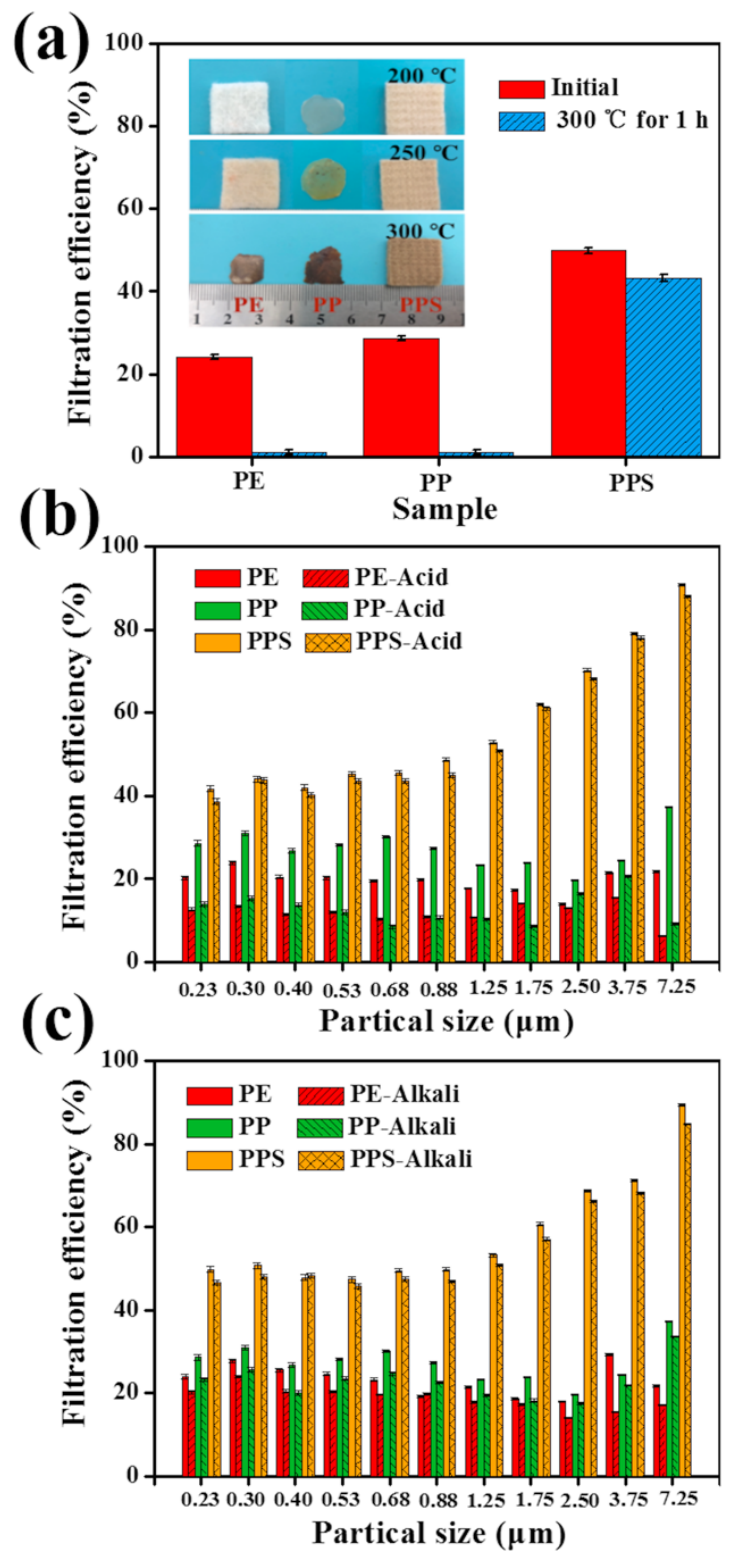

Figure S8. Filtration efficiency of commercial PP, PE and PPS filter materials before and after $300{ }^{\circ} \mathrm{C}(\mathrm{a})$, acid (b) and alkali (c) treatment for $1 \mathrm{~h}$. 


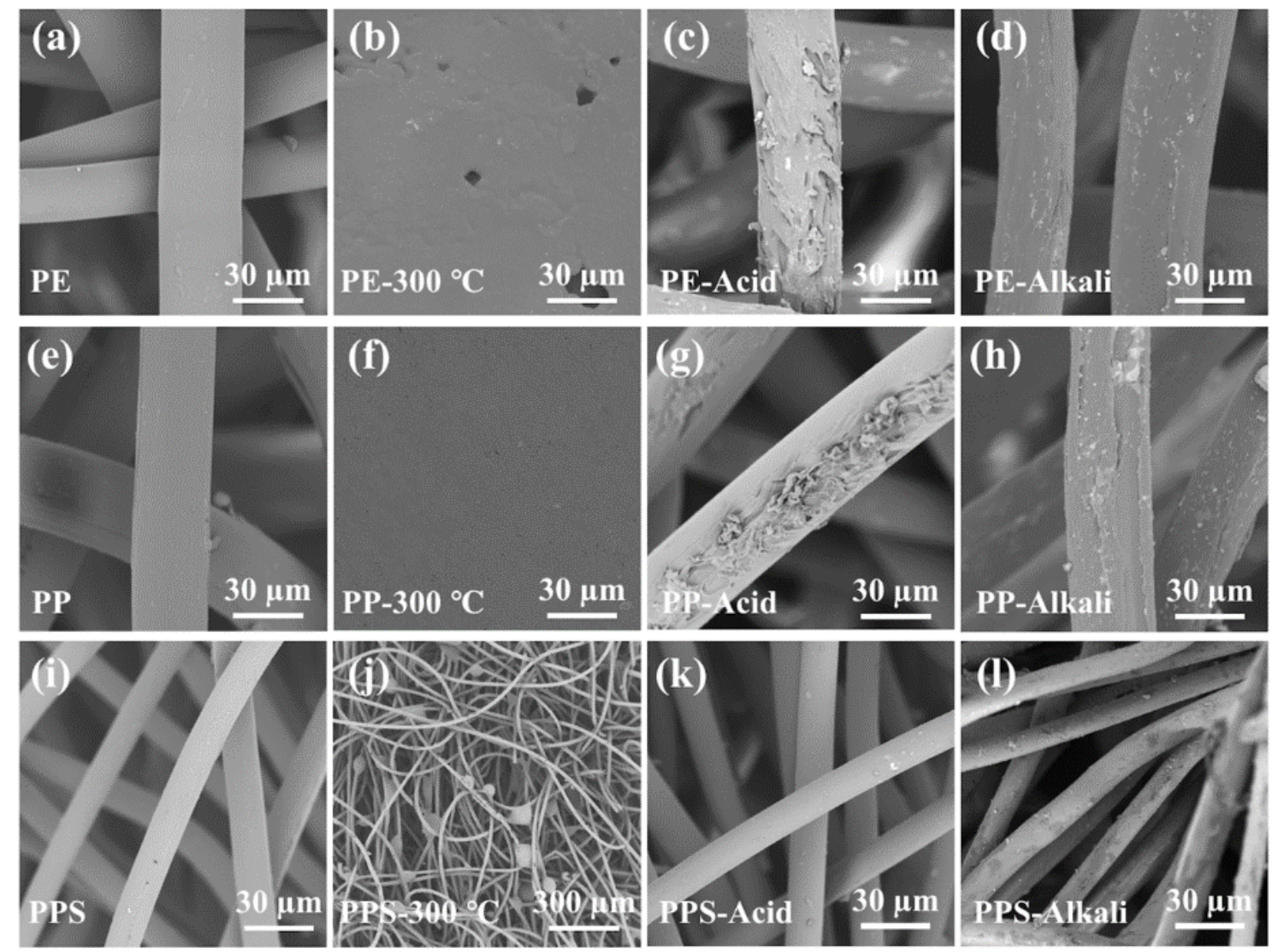

Figure S9. SEM of PE, PP and PPS needle-punched filters (a, e, and i) and after 300 ${ }^{\circ} \mathrm{C}(\mathrm{b}, \mathrm{f}$, and $\mathrm{j})$, acid (c, g, and k) and alkali (d, h, and l) treatments for $1 \mathrm{~h}$. 